\title{
Nucleocytoplasmic shuttling of BID is involved in regulating its activities in the DNA-damage response
}

\author{
G Oberkovitz ${ }^{1}$, L Regev ${ }^{1}$ and A Gross ${ }^{\star, 1}$
}

\begin{abstract}
The BH3-only BID protein acts as a sentinel to interconnect specific death signals to the core apoptotic pathway. Our previous data demonstrated that BID is important for both S-phase arrest and cell death following DNA damage, and that the cell cycle arrest function is regulated by its phosphorylation by the ATM kinase. We also showed that a portion of cellular BID localizes to the nucleus. Here, we demonstrate that etoposide and ionizing radiation induce the exit of BID from the nucleus and that leptomycin B, a specific inhibitor of the nuclear export receptor CRM1, prevents the nuclear exit of BID. BID carries a nuclear export signal (NES) consensus motif; however, it does not seem to be functional. To examine the importance of BID nuclear export, we targeted BID to the nucleus by fusing it to a strong nuclear localization signal (NLS). NLS-BID is phosphorylated in a similar time course as wild-type BID, but does not exit the nucleus following etoposide treatment. Importantly, introducing NLSBID into BID ${ }^{-1-}$ cells failed to restore S-phase arrest and cell death in response to etoposide. These results implicate BID as a nuclear protein and raise the possibility that nucleocytoplasmic shuttling of BID is involved in regulating its activities in the DNAdamage response.
\end{abstract}

Cell Death and Differentiation (2007) 14, 1628-1634; doi:10.1038/sj.cdd.4402181; published online 22 June 2007

Programmed cell death or apoptosis is critical for both the development and maintenance of tissues. Caspases, a family of cysteine proteases, are the major executioners of the apoptotic process, ${ }^{1}$ whereas the BCL-2 family members are the major regulators of this process. ${ }^{2}$ The cell death regulatory mechanisms of the BCL-2 proteins are largely unknown, although it is thought that their function depends mostly on their ability to modulate the release of proteins from the intermembrane space of the mitochondria. The BCL-2 family members are localized at multiple subcellular locations, that is, in the cytosol, nuclear outer membrane, endoplasmic reticulum membrane and mitochondrial outer membrane. In healthy cells, anti-apoptotic BCL-2 family members are mostly found associated with internal membranes, ${ }^{3,4}$ the multidomain pro-apoptotic proteins are localized either at membranes (e.g. BAK $^{5}$ ) or in the cytoplasm (e.g. $\mathrm{BAX}^{6,7}$ ), and the pro-apoptotic $\mathrm{BH}$-only proteins (see below) are predominantly found in the cytosol (e.g. $\left.B A D^{8}\right)$ or associated with the cytoskeleton (e.g. $\mathrm{BIM}^{9}$ ). In response to an apoptotic signal, the pro-apoptotic proteins undergo a conformational change that enables them to target and integrate into membranes, especially the mitochondrial outer membrane, to trigger apoptosis. $^{10}$

An important subset of the pro-apoptotic BCL-2 family members are the BH3-only proteins, such as BID, which act as sensors of intracellular damage. ${ }^{11}$ These proteins are held in check by diverse mechanisms, seemingly at cellular locations at which they can sense specific damage, and once activated, translocate to the mitochondria to communicate the damage signal. BID is a pivotal executioner of apoptosis in vivo, as it is essential for the TNF $\alpha /$ Fas death receptor pathway and is required to suppress tumorigenesis in the myeloid lineage. ${ }^{12,13}$ BID is believed to be relatively inactive in the cytosol until proteolytically cleaved by caspase-8. Cleavage of cytosolic BID at Asp59 yields a p15 C-terminal truncated fragment (tBID) that translocates to the mitochondria, where it induces the activation of BAX and BAK, resulting in the release of cytochrome $c$ and the activation of caspases. ${ }^{14}$ Although most studies in the field emphasize the importance of BID cleavage to activate it, there are several studies that demonstrate an apoptotic role for full-length BID. ${ }^{15-18}$ In addition, it is widely believed that BID is essential only for the TNF $\alpha /$ Fas death receptor pathway and that mitochondria are the major site for its apoptotic action. In contrast, we previously demonstrated that BID partially localizes to the nucleus in healthy cells, and is important for apoptosis induced by DNA damage. ${ }^{19,20}$

In search for BID's role and mechanism of activation in the DNA-damage pathway, we found that etoposide and ionizing radiation, both of which are known to induce doublestrand breaks in DNA, led to its rapid phosphorylation on two serine residues (S61 and S78). These phosphorylations were mediated by ATM, a nuclear serine-threonine protein kinase, which is absent or inactivated in patients with the genomic instability syndrome ataxia-telangiectasia (A-T). The functional consequences of certain ATM phosphorylation events include activation of cell cycle checkpoints, which result in temporary arrest of cell cycle progression to enable DNA repair. ${ }^{21}$ Strikingly, $B I D^{-1-}$ cells failed to arrest in the $S$ phase of the cell cycle following treatment with etoposide;

\footnotetext{
${ }^{1}$ Department of Biological Regulation, Weizmann Institute of Science, Rehovot, Israel

${ }^{*}$ Corresponding author: A Gross, Department of Biological Regulation, Weizmann Institute of Science, Rehovot 76100, Israel.

Tel.: +972 8 9343656; Fax: +972 8 9344116; E-mail: atan.gross@ weizmann.ac.il

Keywords: BID; DNA damage; nucleus; apoptosis; ATM

Abbreviations: NLS, nuclear localization signal; NES, nuclear export signal; LMB, leptomycin B; MEFs, mouse embryonic fibroblasts; GFP, green fluorescence protein Received 07.8.06; revised 16.5.07; accepted 17.5.07; Edited by C Borner; published online 22.6.07
} 
reintroducing wild-type BID (wtBID) restored arrest. In contrast, introducing a nonphosphorylatable BID mutant did not restore arrest in the $S$ phase, and resulted in an increase in the cellular sensitivity to etoposide-induced apoptosis. These results implicated BID as an ATM effector, and indicated that $\mathrm{BID}$, a molecule that was previously considered to be active only as a pro-apoptotic factor in the TNF $\alpha /$ Fas death receptor pathway at the mitochondria, also plays a pro-survival role important for S-phase arrest in the DNA-damage pathway. ${ }^{19,20,22}$

As mentioned above, a portion of cellular BID localizes to the nucleus in healthy cells and in cells exposed to DNA damage; however, it remained unknown whether this portion plays a role in the DNA-damage pathway. Here, we demonstrate that DNA damage results in the nuclear export of BID, and that fusing BID to a strong nuclear localization signal (NLS) inhibits its nuclear export. Importantly, introducing NLS-BID into $B I D^{-1-}$ cells failed to restore S-phase arrest and cell death in response to DNA damage. These results implicate BID as a nuclear protein and raise the possibility that nucleocytoplasmic shuttling of BID is involved in regulating its activities in the DNA-damage response.

\section{Results}

DNA damage results in the nuclear export of BID. Previously, we demonstrated that a portion of cellular BID is localized to the nucleus. ${ }^{19}$ To assess whether DNA damage induces a change in the cellular location of BID, we have generated a BID-GFP chimeric protein and monitored its cellular location in HeLa cells either left untreated or treated with etoposide. In untreated cells, most of the BID-GFP fluorescence appeared in the nucleus (Figure 1a, top left panel). Forty-five minutes after etoposide treatment, there was an increase in the cytosolic fluorescence of BID-GFP, and at 90 min after treatment there was a further increase in this fluorescence (Figure 1a, top panels and bottom left panel). This pattern of fluoresence remained till $150 \mathrm{~min}$ after etoposide treatment. Similar results were obtained with cells treated with ionizing radiation (IR; Figure 1a, bottom right panel), indicating that BID nuclear exit is a general feature of the DNA-damage response.

The exit of proteins from the nucleus is mostly regulated via the nuclear export receptor CRM1, and therefore, we tested whether leptomycin B (LMB), a specific inhibitor of the CRM1, ${ }^{23}$ will prevent the nuclear exit of BID. Strikingly, treatment with LMB prevented the nuclear exit of BID-GFP following etoposide treatment (Figure 1b). These results suggest that DNA-damage results in the nuclear export of $\mathrm{BID}$, and that this export is mediated via the export receptor CRM1.

The nuclear export of proteins by CRM1 is mediated via nuclear export signals (NESs). ${ }^{24}$ The fact that LMB inhibited the nuclear exit of BID suggests that BID carries an NES. Examination of the mouse BID sequence for an NES consensus motif revealed that it carries such a motif (aminoacid residues 35-44; Figure 1c). The REV type NES consensus motif (the one found in BID) is comprised of an 8-12 amino-acid stretch, and mutations of the conserved leucines abolishes the nuclear export of proteins carrying this sequence. ${ }^{24}$ To assess whether the nuclear export of BID is regulated by this motif, we mutated all three of the conserved leucines in this motif to alanines (L35A/L38A/L42A). WtBID and BID-L35A/L38A/L42A, both fused to GFP were expressed in HeLa cells and their cellular locations were assessed in the absence or presence of etoposide. These studies showed that mutating this motif did not affect the nuclear exit of BID (data not shown). Thus, the 35-44 aminoacid stretch in BID does not seem to function as an NES, and therefore, BID nuclear export is probably mediated by another NES-carrying protein.

Fusing BID to a strong nuclear localization signal inhibits its nuclear export. To examine the functional importance of BID nuclear export, we attempted to trap it in the nucleus using a strong NLS. ${ }^{25}$ (Notably, examination of the mouse BID sequence indicated that it does not carry a classical NLS). We found that fusing BID-GFP to the NLS of the SV40 large-T antigen, ${ }^{26}$ drove BID-GFP to the nucleus (Figure 2a, left panel). Importantly, etoposide treatment did not result in the nuclear exit of NLS-BID-GFP (Figure 2a, middle panels), suggesting that this chimeric BID protein is trapped in the nucleus.

Previously, we demonstrated by subcellular fractionation that the endogenous BID in mouse embryonic fibroblasts (MEFs) is loosely associated with the nuclear fraction, and that crosslinking (using formaldehyde) is required to preserve this localization. ${ }^{19}$ To confirm that NLS-BID-GFP is localized to the nucleus, we performed subcellular fractionations in the presence of formaldehyde. Indeed, we found that BID-GFP was mostly localized to the cytoplasmic fraction, whereas NLS-BID-GFP partitions equally between the cytoplasmic and nuclear fractions (Figure $2 b$ ). The fact that a significant portion of NLS-BID-GFP appears in the cytoplasmic fraction (in contrast to the results we obtained in the fluorescence studies) suggests that it leaks out of the nucleus during preparation.

\section{Introduction of NLS-BID into $B I D^{-/}$MEFs fails to restore susceptibility to etoposide-induced cell} death. Previously, we demonstrated that $B I D^{-1-}$ MEFs are less susceptible than $B I D^{+/+}$MEFs to DNA-damageinduced cell death, and that reintroduction of wtBID restored susceptibility to DNA damage. ${ }^{17,19}$ To explore the ability of NLS-BID to restore susceptibility to DNA damage, we generated single stable clones of $B I D^{-/-}$MEFs that express either wtBID or NLS-BID, or carry an empty vector. Initially, we confirmed the cellular location of wtBID and NLS-BID in these clones, and showed by both subcellular fractionation and by immunofluoresence that wtBID was mostly cytosolic, whereas NLS-BID partitions equally between the cytoplasmic and nuclear compartments (Figure 3a). Next, we assessed the levels of etoposideinduced cell death in four of wtBID, NLS-BID and empty vector stable clones each. We found that the wtBID clones showed a two- to threefold higher level of cell death compared to the empty vector clones, whereas there was no significant difference in the levels of cell death between the NLS-BID clones and the empty vector clones (Figure 3b; 
a Etop (min):
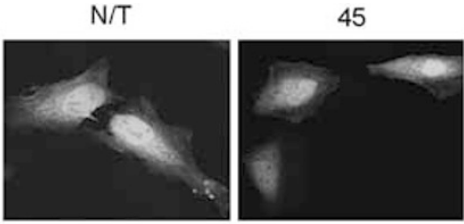

Etop

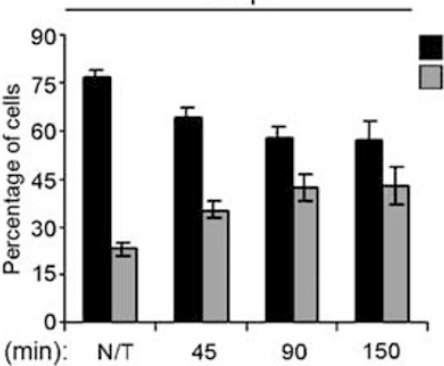

90

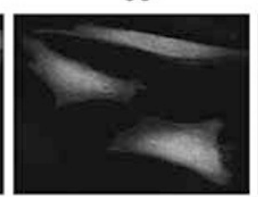

IR

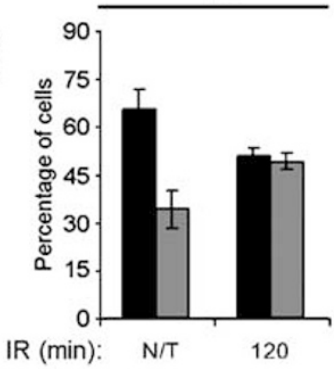

150
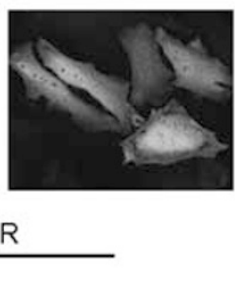

b Etop (min):

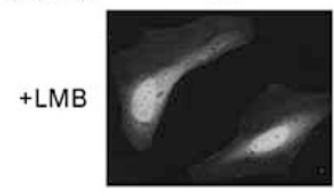

45

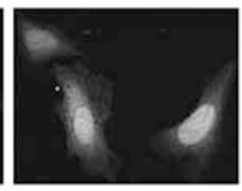

90

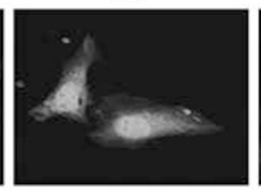

150
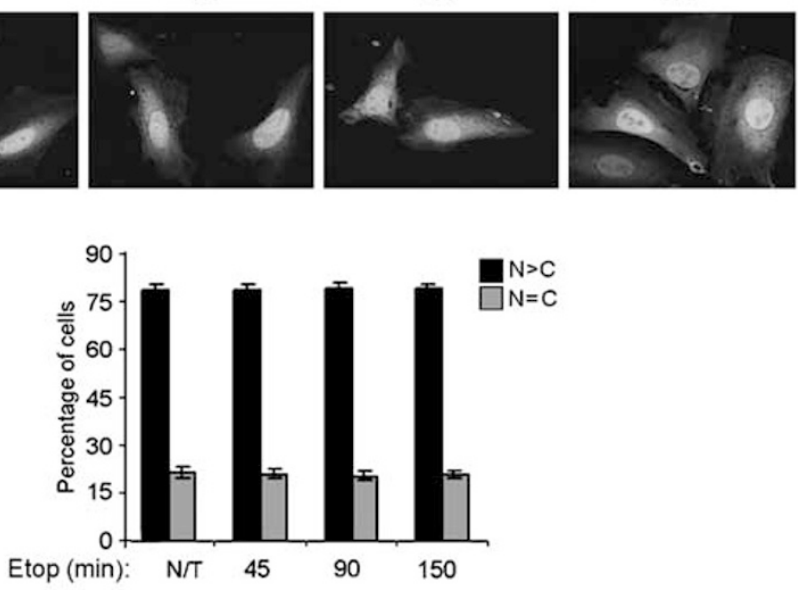

c

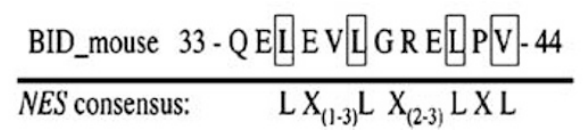

Figure 1 DNA damage results in the nuclear export of BID. (a) DNA damage results in the nuclear exit of BID-GFP. HeLa cells were grown on coverslips and transfected with a BID-GFP construct. At $16 \mathrm{~h}$ post-transfection, the cells were either left untreated (N/T) or treated with etoposide (100 $\mu \mathrm{M})$ or IR (50 Gy). At the indicated times postetoposide or -IR treatment, the cells were fixed and pictures were taken using a confocal microscope (top panels show only etoposide-treated cells). Bottom: the histograms represent the percentage of cells out of transfected cells that show a prevalence of BID-GFP in the nucleus $(N>C)$ or an equal distribution of BID-GFP between nucleus and cytoplasm $(\mathrm{N}=\mathrm{C})$. The data are expressed as the mean \pm S.E.M. of three independent experiments in which 100 cells were analyzed each time. The left panel represents cells treated with etoposide, and the right panel represents cells treated with IR. (b) LMB prevents the nuclear exit of BID-GFP. HeLa cells were treated as above. At $16 \mathrm{~h}$ posttransfection, the cells were pre-treated for $1 \mathrm{~h}$ with LMB $(5 \mathrm{ng} / \mu \mathrm{l})$, and then either left untreated (N/T) or treated with $100 \mu \mathrm{M}$ etoposide. At the indicated times after etoposide treatment, the cells were fixed and pictures were taken using a confocal microscope (top panels). The histograms shown in the bottom panel were generated as described in (a). (c) Mouse BID carries an NES consensus motif. The NES consensus motif found in BID is shown in the top line, and in the bottom line appears the general NES consensus motif that contains four closely spaced leucines, which can be substituted by other large hydrophobic residues (Isoleucines/Valines)

only two clones from each are shown). Western blot analysis using anti-BID antibodies indicated that the decreased levels of cell death seen in the NLS-BID clones in response to etoposide was not due to lower levels of expression of NLS-BID (Figure 3c). Thus, trapping BID in the nucleus impairs its cell death activity in response to DNA damage.

$B I D^{-/-}$MEFs expressing NLS-BID fail to arrest in the S phase of the cell cycle following etoposide treatment. Previously, we demonstrated that BID is important for S-phase arrest following DNA damage, and that this novel activity of BID is regulated by its phosphorylation by ATM. ${ }^{19}$ To explore the effect of NLS-BID on S-phase arrest, we performed cell cycle analysis on four wtBID and on four NLS-BID clones either untreated or treated with etoposide. Surprisingly, etoposide treatment did not induce S-phase arrest in the NLS-BID clones and cells from these clones rapidly accumulated in the G2 phase, whereas cells from the wtBID clones accumulated in the $S$ phase (Figure 4a; only two clones from each are shown).

To exclude the possibility that the stable clones expressing NLS-BID may have developed a compensating genetic or 
extragenetic change that is responsible for the observed effects, we performed cell cycle experiments with $B I D^{-1-}$ MEFs 3 days post-infection with either wtBID or NLS-BID. We found that $B I D^{-1-}$ cells infected with wtBID showed an $\sim 10 \%$ increase in $S$ phase following etoposide treatment, whereas $B I D^{-/-}$cells infected with NLS-BID did not (Supplementary Figure 1). These results confirm that NLS-BID is indeed impaired in its ability to induce S-phase arrest, as it is unlikely that 3 days post-infection the NLS-BID cells developed a compensating genetic change that is responsible for the observed effects.

To assess whether the impaired ability to arrest in S phase was due to an inability of NLS-BID to be phosphorylated in response to etoposide treatment, wIBID and NLS-BID cells
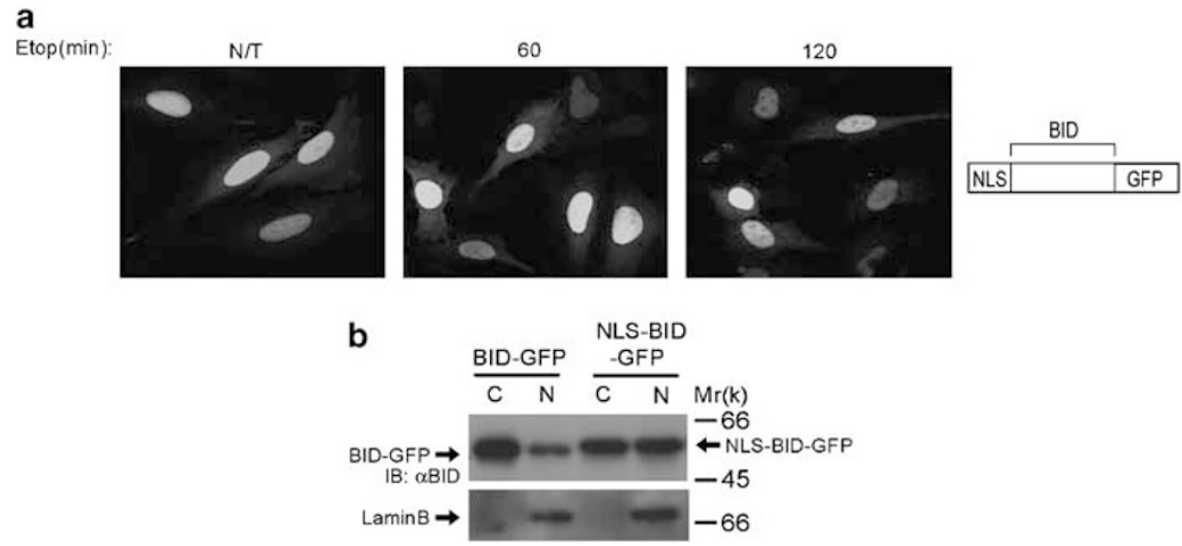

Figure 2 Fusing BID to a strong NLS inhibits its nuclear export. (a) NLS-BID-GFP does not exit the nucleus in response to etoposide. HeLa cells were transfected with NLS-BID-GFP, and $16 \mathrm{~h}$ post-transfection, the cells were either left untreated (N/T) or treated with $100 \mu \mathrm{M}$ etoposide. At the indicated times after etoposide treatment, the cells were fixed and pictures were taken using a confocal microscope (left and middle panels). Right panel: a schematic representation of the NLS-BID-GFP protein. (b) NLS-BID-GFP is localized to the nucleus. HeLa cells were transfected with either BID-GFP or NLS-BID-GFP, and $16 \mathrm{~h}$ post-transfection, the cells were treated with formaldehyde and subfractionated. Aliquots of the cytoplasmic $(\mathrm{C})$ and nuclear $(\mathrm{N})$ fractions were subjected to SDS-PAGE followed by Western blot analysis using anti-BID Abs (top). The blot was stripped and reprobed with anti-lamin B Abs to mark the nuclear fraction (bottom)

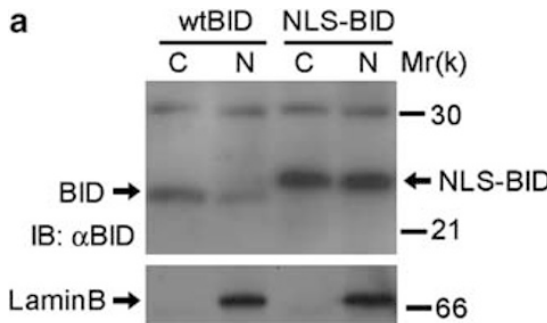

b

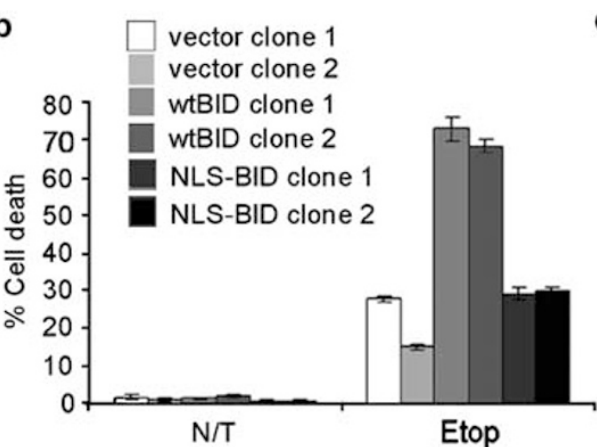

wtBID

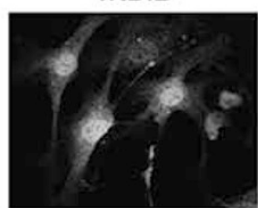

IF: $\alpha \mathrm{BID}$
C

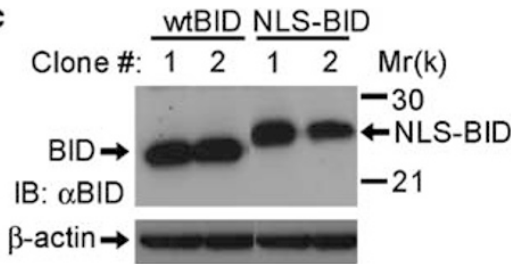

Figure 3 Introduction of NLS-BID into BID ${ }^{-/-}$MEFs fails to restore susceptibility to etoposide-induced cell death. (a) NLS-BID is localized to the nucleus in the MEFstable clones. Left panel: BID ${ }^{-1}$ MEFs stably expressing either wtBID or NLS-BID (one clone from each) were treated with formaldehyde and subfractionated. Aliquots of the cytoplasmic (C) and nuclear (N) fractions were subjected to SDS-PAGE followed by Western blot analysis using anti-BID Abs (top). The blot was stripped and reprobed with anti-lamin B Abs to mark the nuclear fraction (bottom). Right panels: BID ${ }^{-/-}$MEFs stably expressing either wtBID or NLS-BID (one clone from each) were fixed and stained with anti-BID Abs, and pictures were taken using a confocal microscope. (b) BID ${ }^{-1-}$ MEFs stably expressing either wtBID or NLS-BID, or carrying an empty vector (two clones from each) were treated with $50 \mu \mathrm{M}$ etoposide for $24 \mathrm{~h}$, and cell death was monitored by FACScan using PI dye exclusion. The data represent the means \pm S.E.M. of pooled results from three independent experiments. (c) The decreased death obtained with the NLS-BID clones is not due to lower levels of expression of NLS-BID. The two wtBID and two NLS-BID clones were lysed, and equal amounts of protein (20 $\mu \mathrm{g}$ per lane) were subjected to SDS-PAGE, followed by Western blot analysis using anti-BID Abs (top). The blot was stripped and reprobed with $\beta$-actin Abs to control for loading (bottom) 

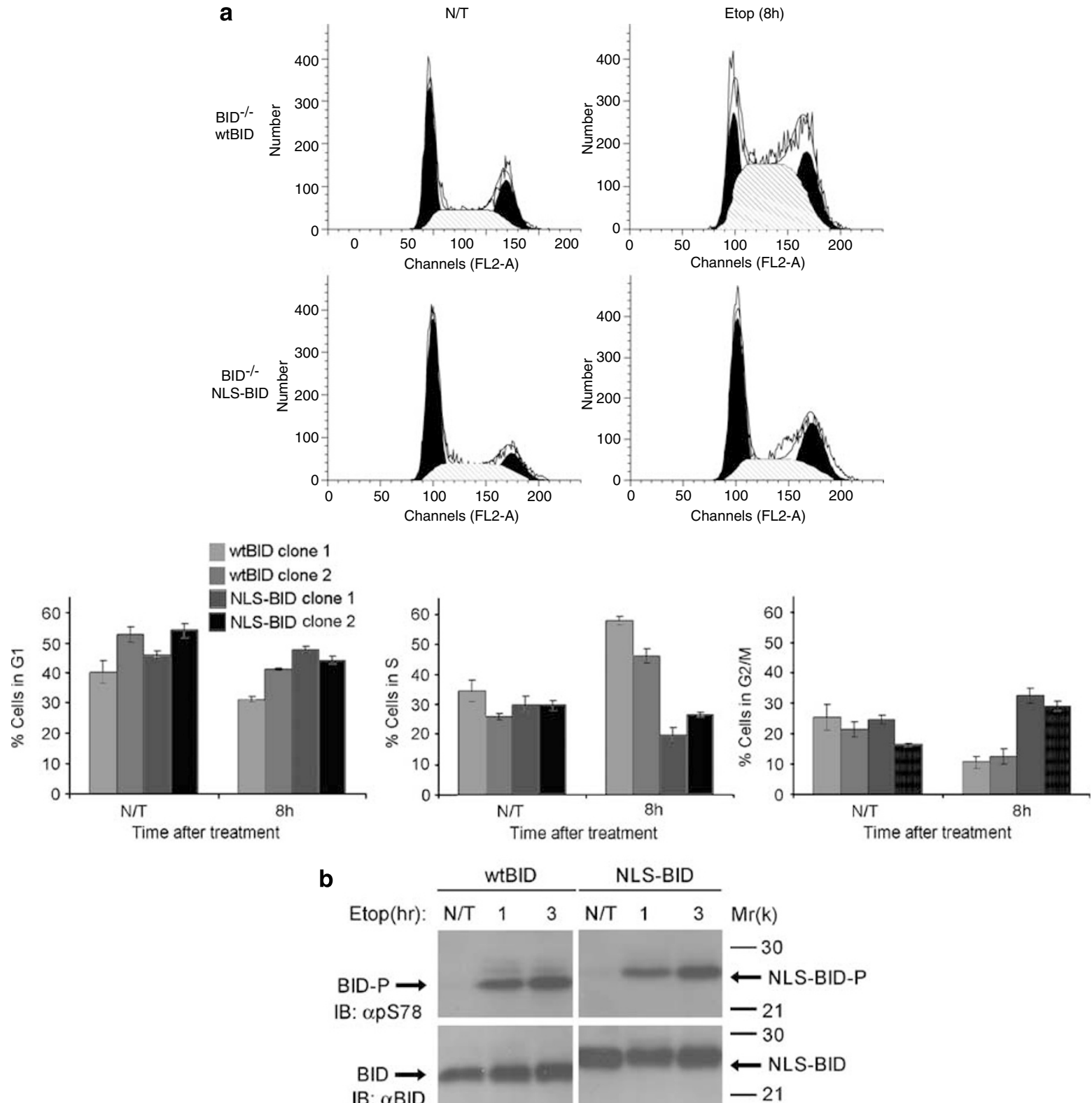

Figure $4 B I D^{-/-}$MEFs expressing NLS-BID fail to arrest in the S phase of the cell cycle following etoposide treatment. (a) BID ${ }^{-/}$MEFs stably expressing either wtBID or NLS-BID (the same four clones shown in Figure 3b) were either left untreated (N/T) or treated with $10 \mu \mathrm{M}$ etoposide, and $8 \mathrm{~h}$ post-treatment, the DNA content was analyzed by flow cytometry. The actual raw data from a representative experiment together with multiline plots generated by the ModFit LT computer software program are show in the top panel. The dark histograms represent the percent of cells in the G1 and G2/M phases, and the hatched histograms represent the percent of cells in S phase. The exact percentage of cells in each phase of the cell cycle (in each of the four clones) is shown in the bottom three panels. The data represent the means + S.E.M. of pooled results from three independent experiments. (b) NLS-BID is phosphorylated on S78 in a similar time course as wtBID. BID ${ }^{-/-}$MEFs stably expressing either wtBID or NLS-BID (one clone from each) were either left untreated (N/T) or treated with $50 \mu \mathrm{M}$ etoposide. At the indicated time points, the cells were lysed and equal amounts of protein $(20 \mu \mathrm{g}$ per lane) were subjected to SDS-PAGE, followed by Western blot analysis using either anti-pS78 Abs (top) or antibodies to regular BID (bottom)

were treated with etoposide and Western blot analyzed using the phosphospecific antibodies to phosphoserine $78 .{ }^{19}$ Figure $4 \mathrm{~b}$ shows that NLS-BID is phosphorylated in a similar time course as wtBID, suggesting that the inability of NLS-BID to induce S-phase arrest is not due to impaired phosphorylation.

Taken together, these results suggest that nuclear export of BID is important for its S-phase arrest function in the DNA-damage response.

\section{Discussion}

In the present study, we demonstrate that DNA damage induces the exit of BID from the nucleus and that this exit is mediated via the nuclear export receptor CRM1. We also show that fusing BID to a strong NLS inhibits its nuclear export. Importantly, NLS-BID is phosphorylated by ATM in response to DNA damage, but is impaired in its ability to induce S-phase arrest and to sensitize cells to DNA-damage-induced cell death. 
As described in the introductory text, the $\mathrm{BH}$-only proteins are sentinels of intracellular damage. These proteins are held in check by diverse mechanisms and seemingly at cellular locations in which they can sense or communicate specific damage. Previously, we demonstrated that a portion of BID localizes to the nucleus. ${ }^{19}$ As the majority of currently identified substrates of ATM are nuclear proteins, it is most likely that the nuclear portion of the BID molecules is phosphorylated by ATM. Our results showing that NLS-BID is phosphorylated in response to DNA damage (Figure 4), further argue that BID is indeed phosphorylated by ATM in the nucleus. If this is indeed the case then BID might function as a sentinel of DNA damage by being embedded as an integral participant in the pathway that it monitors.

Where in the cell is BID executing its function? Many of the ATM substrates are phosphorylated at the damaged sites, and some of them are rapidly distributed to the undamaged parts of the nucleus (and perhaps to other cellular locations) to reach their physiological targets. ${ }^{27}$ Here, we demonstrate that DNA damage results in the nuclear export of BID (Figure 1), and that trapping BID in the nucleus inhibits its functions in the DNA-damage response (Figures 3 and 4). Taken together, these results suggest that BID is required to exit the nucleus to execute its functions in this pathway. Thus, we suspect that BID is phosphorylated at the damaged sites and rapidly distributed to another cellular location (outside of the nucleus) to execute its functions. One attractive location is the mitochondria, as several $\mathrm{BH}$-only proteins are known to communicate specific damage by translocating from the site of damage to the mitochondria. Notably, a collaborating group has previously published that DNA damage induces the translocation of cytoplasmic BID to the nucleus. ${ }^{20} \mathrm{~A}$ possible explanation for this discrepancy is that BID is moving both into and out of the nucleus, and that in our setting, we are detecting its net exit. If DNA damage indeed results in the translocation of cytoplasmic BID to the nucleus (translocation of the nonphosphorylated form), it remains to be determined the functional importance of this translocation.

Do our findings using BID-GFP apply to endogenous BID? We have previously reported, based on immunofluoresence studies, that a portion of endogenous BID is localized to the nucleus. ${ }^{19}$ However, we did not detect a change in the cellular location of endogenous BID following etoposide treatment, as we did using BID-GFP in this study. A possible explanation for these differences is that it is more difficult to detect changes in the cellular location of proteins using immunofluoresence, as compared to using GFP fusion proteins. In the case of BID, it is even more difficult as we detected only an $\sim 20 \%$ increase in cytosolic BID-GFP following DNA damage (Figure 1).

We demonstrated that $L M B$ prevents the nuclear exit of BID (Figure 1), suggesting that this exit occurs via the nuclear export receptor CRM1. On the other hand, mutating the NES consensus motif found in BID had no effect on its nuclear export, suggesting that this motif does not function as a NES. As the export of nuclear proteins by CRM1 is mediated via NESs, ${ }^{24}$ it is likely that the nuclear export of BID is mediated by another NES-carrying protein. Recently, such a mechanism has been demonstrated by showing that nucleophosmin (NPM), which utilizes a conserved CRM1-dependent NES to enable its nucleocytoplasmic shuttling, provides a necessary chaperoning activity for the nuclear export of ribosomal protein L5 (rpL5). ${ }^{28}$ Finally, it is tempting to speculate that ATM-mediated phosphorylation of BID regulates its nuclear export. However, this does not seem to be the case, as DNA damage resulted in the nuclear exit of the nonphosphorylatable BID mutant (BID-S61A/S78A-GFP; data not shown).

How does BID induce S-phase arrest? One possible mechanism for inducing cell cycle arrest would be that phosphorylated BID directly interacts with a cell cycle regulatory protein located in the nucleus to directly halt cell cycle progression at the S-phase checkpoint. However, based on our current results, it is equally possible that BID affects cell cycle progression indirectly by interacting with a 'non-cell cycle' protein that could be located outside of the nucleus. BID is also involved in promoting cell death following DNA damage, ${ }^{17,19}$ and it is well established that BID initiates death at the mitochondria. Thus, it is not surprising that trapping BID in the nucleus inhibits its ability to sensitize cells to DNA damage-induced death (Figure 3).

Finally, are caspases involved in the nucleocytoplasmic shuttling of BID? We monitored the cellular location of BIDGFP in HeLa cells in the presence of the broad caspase inhibitor zVAD-fmk, and found that inhibition of caspases had no affect on the nuclear export of BID following DNA damage. Moreover, we performed similar studies with the caspase-8 noncleavable mutant of BID (BID-D59A-GFP), and found that DNA damage resulted in the nuclear exit of this mutant. We also used zVAD-fmk to test whether the S-phase arrest might be an indirect consequence of cell death rather than a direct effect of BID nuclear exit. We found that inhibition of caspases did not affect the ability of the wtBID cells to arrest in the $S$ phase and did not change the differences in the cell cycle distribution between the wtBID and the NLS-BID cells (data not shown). Thus, caspases do not seem to be involved in the nuclear exit of BID or in the ability of BID to induce S-phase arrest.

In summary, this study raises the novel possibility that BID, a molecule that was previously considered to be active only at the mitochondria, may also be active in the nucleus and that its activity in the DNA-damage pathway requires nuclear export. If BID is indeed acting in such a manner, then it is an excellent candidate to communicate DNA damage/repair processes to other cellular compartments.

\section{Materials and Methods}

Constructs. For the construction of the NLS-BID expression vector, BID was PCR amplified, using a $5^{\prime}$ primer consisting of a BamHI restriction site followed by the SV40-LT-NLS, and BID's first 26 bases, and a $3^{\prime}$ primer consisting of an EcoRI restriction site, followed by BID's $C$ terminus antisense strand. $5^{\prime}$ primer: GCAGGATCCATGCCGAAGAAGAAGCGCAAGGTAGACTCTGAGGTCAGCAAC GGTTCCGGC. 3' primer: GCGAATTCGTCAGTCCATCTCGTTTCTAACCAAGTT CCT. To introduce point mutations into the NES consensus motif of BID, we have used the QuickChange ${ }^{\mathrm{TM}}$ site directed mutagenesis kit (Stratagene) using the $5^{\prime}$ primer: CTGTACTCGCCAAGAGGCGGAGGTGGCGGGTCGGGAAGCGCCTGTGCAAG CTTAC and the $3^{\prime}$ primer: GTAAGCTTGCACAGGCGCTTCCCGACCCGCCA CCTCCGCCTCTTGGCGAGTACAG. The fragments were digested and ligated into the pcDNAlll vector (Invitrogen). For the construction of BID-GFP, BID-L35A/L38A/ L42A-GFP, BID-D59A-GFP and BID-S61A/S78A-GFP, BID was PCR amplified using a $5^{\prime}$ primer consisting of a Xhol restriction site, followed by BID's $\mathrm{N}$ terminus sense strand and a $3^{\prime}$ primer consisting of an EcoRI restriction site, followed by BID's $C$ terminus antisense strand. For the construction of NLS-BID-GFP, BID was PCR amplified, using a $5^{\prime}$ primer consisting of an Xhol restriction site, followed by 
the SV40-LT-NLS and a $3^{\prime}$ primer consisting of an EcoRI restriction site, followed by BID's $C$ terminus antisense strand. $5^{\prime}$ primers: CCGCTCGAGATGGACTCTGAGGT CAGCAACG; CCGCTCGAGATGCCGAAGAAGAAGCGCAAG and the $3^{\prime}$ primer: GCGAATTCGGTCCATCTCGTTTC. The fragments were digested and ligated into the pEGFP-N1 vector (Clontech).

Human cell lines and transient transfection. HeLa, a human cervical adenocarcinoma cell line, was maintained in $10 \%$ fetal bovine serum. Transient transfections were performed using lipofectamine 2000 (Gibco BRL).

Generation of $\mathrm{BID}^{-/-}$MEF stable clones expressing wtBID or NLS-BID. BID ${ }^{-1-}$ MEF stable clones expressing either wtBID or NLS-BID, or carrying an empty vector were generated as described previously. ${ }^{19}$

Viewing the cellular location of GFP fusion proteins. HeLa cells were grown on glass coverslips, transfected with either of the GFP constructs, treated as indicated and fixed with $4 \%$ paraformaldehyde in PBS for $10 \mathrm{~min}$. The coverslips were mounted and confocal microscopy was performed by using an Axiovert 100 TV microscope (Zeiss, Oberkochen, Germany) attached to the BioRad Radiance 2000 laser scanning system (Bio-Rad) and operated by LaserSharp software.

Immunofluorescence. For immunofluorescence experiments, cells were grown on glass coverslips and fixed with $4 \%$ paraformaldehyde in PBS for 10 min and permeabilized with $0.2 \%$ Triton X-100 in PBS for $5 \mathrm{~min}$. For blocking, the cells were incubated in PBS containing $0.1 \%$ Triton and $3 \%$ BSA for $1 \mathrm{~h}$ at room temperature. For immunostaining, cells were incubated for $2 \mathrm{~h}$ at room temperature with anti-murine BID Abs in blocking solution. After three washes with PBS containing $0.1 \%$ Triton, the cells were stained for $1 \mathrm{~h}$ at room temperature with $\mathrm{Cy}^{\mathrm{TM}}$ 2-conjugated AffiniPure donkey anti-rabbit Abs (dilution 1:300, Jackson ImmunoResearch). After three additional washes with PBS containing $0.1 \%$ Triton, the coverslips were mounted and confocal microscopy was performed as described above.

Cell viability assays. MEFs were treated with $50 \mu \mathrm{M}$ etoposide for $24 \mathrm{~h}$. Cell viability was determined by propidium iodide (PI) dye exclusion. PI $(25 \mu \mathrm{g} / \mathrm{ml})$ was added to the cells immediately before analysis by FACScan (Beckton Dickinson).

Cell cycle assays. MEFs were treated with $10 \mu \mathrm{M}$ etoposide for $8 \mathrm{~h}$, and then collected for fixation in methanol. Following fixation, cells were washed and resuspended in PBS with $25 \mu \mathrm{g} / \mathrm{ml} \mathrm{PI}$ and $50 \mu \mathrm{g} / \mathrm{ml} \mathrm{RNAse} \mathrm{a} 0.5 \mathrm{~h}$ before FACScan analysis. Analysis of the cell cycle results was performed using the ModFit LT program. ${ }^{29}$

Formaldehyde treatment and subcellular fractionation. Formaldehyde treatment and subcellular fractionations were performed as described previously. ${ }^{19}$

Western blot analysis and antibodies. Proteins were size-fractionated by SDS-PAGE and then transferred to PVDF membranes (Bio-Rad). Western blots were developed by use of the enhanced chemiluminescence reagent (Amersham). Polyclonal anti-murine BID Abs, ${ }^{19}$ anti-pS78 Abs, ${ }^{19}$ anti-lamin B Abs (Santa Cruz) and anti- $\beta$ actin Abs (Santa Cruz) were used for Western blotting.

Acknowledgements. This work was supported, in part, by the Israel Science Foundation, the Israel Cancer Association (through a donation from Teva Pharmaceutical Industries Ltd.), Ataxia-Telangiectasia Foundation (A-TCP), Minerva Stiftung, MDM ICR Research Award, the Willner Family Center for Vascular Biology and Mr and Mrs Stanley Chais. A.G. is the incumbent of the Armour Family Career Development Chair of Cancer Research.
1. Shi Y. Mechanisms of caspase activation and inhibition during apoptosis. Mol Cell 2002; 9 : 459-470.

2. Danial NN, Korsmeyer SJ. Cell death: critical control points. Cell 2004; 116: 205-219.

3. Hockenbery D, Nunez G, Milliman C, Schreiber RD, Korsmeyer SJ. Bcl-2 is an inner mitochondrial membrane protein that blocks programmed cell death. Nature 1990; 348: 334-336

4. Krajewski S, Tanaka S, Takayama S, Schibler MJ, Fenton W, Reed JC. Investigation of the subcellular distribution of the bcl-2 oncoprotein: residence in the nuclear envelope, endoplasmic reticulum, and outer mitochondrial membranes. Cancer Res 1993; 53 4701-4714.

5. Griffiths GJ, Dubrez L, Morgan CP, Jones NA, Whitehouse J, Corfe BM et al. Cell damageinduced conformational changes of the pro-apoptotic protein Bak in vivo precede the onset of apoptosis. J Cell Biol 1999; 144: 903-914.

6. Hsu YT, Wolter KG, Youle RJ. Cytosol-to-membrane redistribution of Bax and Bcl-X(L) during apoptosis. Proc Natl Acad Sci USA 1997; 94: 3668-3672.

7. Gross A, Jockel J, Wei MC, Korsmeyer SJ. Enforced dimerization of BAX results in its translocation. mitochondrial dysfunction and apoptosis. EMBO J 1998; 17: 3878-3885.

8. Zha J, Harada H, Yang E, Jockel J, Korsmeyer SJ. Serine phosphorylation of death agonist $B A D$ in response to survival factor results in binding to 14-3-3 not BCL-X(L). Cell 1996; 87: 619-628.

9. Puthalakath $H$, Huang DC, O'Reilly LA, King SM, Strasser A. The proapoptotic activity of the $\mathrm{Bcl}-2$ family member Bim is regulated by interaction with the dynein motor complex. Mol Cell 1999; 3: 287-296

10. Wang X. The expanding role of mitochondria in apoptosis. Genes Dev 2001; 15 2922-2933

11. Willis SN, Adams JM. Life in the balance: how BH3-only proteins induce apoptosis. Curr Opin Cell Biol 2005; 17: 617-625.

12. Yin XM, Wang K, Gross A, Zhao Y, Zinkel S, Klocke B et al. Bid-deficient mice are resistant to Fas-induced hepatocellular apoptosis. Nature 1999; 400: 886-891.

13. Zinkel SS, Ong CC, Ferguson DO, Iwasaki H, Akashi K, Bronson RT et al. Proapoptotic $\mathrm{BID}$ is required for myeloid homeostasis and tumor suppression. Genes Dev 2003; 17 229-239.

14. Gross A, McDonnell JM, Korsmeyer SJ. BCL-2 family members and the mitochondria in apoptosis. Genes Dev 1999; 13: 1899-1911.

15. Desagher S, Osen-Sand A, Nichols A, Eskes R, Montessuit S, Lauper S et al. Bid-induced conformational change of $\mathrm{Bax}$ is responsible for mitochondrial cytochrome $\mathrm{c}$ release during apoptosis. J Cell Biol 1999; 144: 891-901.

16. Tafani M, Karpinich NO, Hurster KA, Pastorino JG, Schneider T, Russo MA et al. Cytochrome $c$ release upon Fas receptor activation depends on translocation of full-length bid and the induction of the mitochondrial permeability transition. J Biol Chem 2002; 277 10073-10082.

17. Sarig R, Zaltsman Y, Marcellus RC, Flavell R, Mak TW, Gross A. BID-D59A is a potent inducer of apoptosis in primary embryonic fibroblasts. J Biol Chem 2003; 278: 10707-10715.

18. Valentijn AJ, Gilmore AP. Translocation of full-length Bid to mitochondria during anoikis. J Biol Chem 2004; 279: 32848-32857.

19. Kamer I, Sarig R, Zaltsman Y, Niv H, Oberkovitz G, Regev L et al. Proapoptotic BID is an ATM effector in the DNA-damage response. Cell 2005; 122: 593-603.

20. Zinkel SS, Hurov KE, Ong C, Abtahi FM, Gross A, Korsmeyer SJ. A role for proapoptotic BID in the DNA-damage response. Cell 2005; 122: 579-591.

21. Shiloh Y. The ATM-mediated DNA-damage response: taking shape. Trends Biochem Sci 2006; 31: 402-410.

22. Gross A. BID as a double agent in cell life and death. Cell Cycle 2006; 5: 582-584

23. Wolff B, Sanglier JJ, Wang Y. Leptomycin B is an inhibitor of nuclear export: inhibition of nucleo-cytoplasmic translocation of the human immunodeficiency virus type 1 (HIV-1) Rev protein and Rev-dependent mRNA. Chem Biol 1997; 4: 139-147.

24. Henderson BR, Eleftheriou A. A comparison of the activity, sequence specificity, and CRM1-dependence of different nuclear export signals. Exp Cell Res 2000; 256: 213-224.

25. Nigg EA. Nucleocytoplasmic transport: signals, mechanisms and regulation. Nature 1997; 386: 779-787.

26. Kalderon D, Richardson WD, Markham AF, Smith AE. Sequence requirements for nuclear location of simian virus 40 large-T antigen. Nature 1984; 311: 33-38.

27. Lukas C, Falck J, Bartkova J, Bartek J, Lukas J. Distinct spatiotemporal dynamics of mammalian checkpoint regulators induced by DNA damage. Nat Cell Biol 2003; 5: 255-260.

28. Yu Y, Maggi Jr LB, Brady SN, Apicelli AJ, Dai MS, Lu H et al. Nucleophosmin is essential for ribosomal protein L5 nuclear export. Mol Cell Biol 2006; 26: 3798-3809.

29. Tripathi AK, Chaturvedi R, Ahmad R, Asim M, Sawlani KK, Singh MK et al. Flow cytometric analysis of aneuploidy and S-phase fraction in chronic myeloid leukemia patients: role in early detection of accelerated phase. Leuk Res 2003; 27: 899-902. 